\title{
MAGNETIC ENERGY CONVERSION IN THE CORONA AND MAGNETOSPHERE
}

\author{
GERHARD HAERENDEL \\ Max-Planck-Institut für extraterrestrische Physik, \\ 85740 Garching, Germany
}

Five different categories of magnetic energy conversion will be discussed, and their manifestations in the ionosphere-magnetosphere and chromosphere - corona will be compared. They are: (1) Ohmic dissipation of d.c. currents, (2) Damping of Alfvén waves, (3) Magnetic pumping, (4) Reconnection, and (5) Electrostatic acceleration parallel B. Manifestations are: (1) Ionospheric heating by Pedersen currents and generation of chromospheric faculae and plages by dissipation of field-aligned currents; (2) Generation of spicules by collisional damping of Alfvén -waves and Alfvén-wave damping in the corona by resonant absorption; (3) Drift resonance acceleration of radiation belt particles and stochastic acceleration of high-energy flare particles by Alfvén waves; (4) Reconnection at the magnetopause and in the tail, plasmoid formation and a wide variety of configuration changes and mhd instabilities in the corona for which reconnection plays a decisive role; (5) Acceleration by field-aligned potential drops as origin of auroral arcs and of $>10 \mathrm{MeV}$ electron and ion beams in solar flares. Some of these mechanisms, e.g. plages, spicules, nanoflares and field-aligned electrostatic accelerations will be selected for more detailed discussion. 